\title{
Perceived Usefulness of Telehealth Among Rural Medical Providers: Barriers to Use and Associations with Provider Confidence
}

\author{
Danielle L. Terry ${ }^{1}\left[\right.$ [D Shirley P. Buntoro ${ }^{1}$
}

Received: 19 August 2020 / Revised: 14 May 2021 / Accepted: 28 May 2021 / Published online: 5 June 2021

(c) The Author(s), under exclusive licence to Springer Nature Switzerland AG 2021

\begin{abstract}
Objective Telehealth has been identified as an efficient and safe way of increasing access to healthcare during the COVID19 pandemic. Understanding providers' perceptions of telehealth usage in rural communities may help other communities understand barriers and concerns related to implementation, during and post-pandemic. This study aimed to (a) examine rates of telemedicine use among rural providers, (b) determine whether changes in telehealth use in this group were associated with provider confidence and perceived usefulness of technology, (c) compare these providers' perceptions of the "usefulness" of technology prior to and during the COVID-19 pandemic, and (d) examine barriers to implementation and use of telehealth within a rural sample.

Method Six-hundred eighty-six medical providers working at a rural Pennsylvania teaching hospital and associated satellite clinics were surveyed anonymously. Surveys included the Perceived Usefulness of Technology Scale and questions to identify barriers that prohibited the use of telehealth.

Findings/Results Of 136 respondents, $86 \%$ reported no prior experience using virtual technology for patient encounters. Use of telehealth care increased by $34 \%$ following the pandemic. Provider confidence in his/her/their abilities was positively associated with increased use of telehealth and perceived usefulness of technology. Provider-identified barriers to implementation included necessity of physical exams and lack of technological literacy.

Conclusions Both medical providers and patients continue to face various barriers to seamless integration of care. Devising ways to increase self-confidence and efficacy for use of telehealth among providers might be an additional way to increase telehealth use.
\end{abstract}

Keywords Telehealth $\cdot$ COVID-19 $\cdot$ Rural medicine

The worldwide pandemic and public health concern resulting from a new coronavirus (i.e., COVID-19) has highlighted the need for innovative and alternative approaches to physically distant medical care (Smith et al., 2020). Termed COVID-19, this pandemic has become a significant public health concern, with medical, provider, and resource shortages projected. Nationally, the Centers for Disease Control and Prevention has noted that all 50 states of the USA have had cases of COVID-19, and by now all states have reported community spread of the disease (Centers for Disease Control \& Prevention, 2020).

Danielle L. Terry

danielle.terry@guthrie.org

1 Guthrie Robert Packer Hospital, Saye, PA, USA
Current data suggest that transmission of COVID-19 occurs through respiratory droplets that are produced when an individual talks, sneezes, or coughs. As of May 2021, there have been more than 31 million cases identified and over 570,000 deaths in the USA (Centers for Disease Control $\&$ Prevention, 2020). Key factors in reducing transmission of the disease include maintaining $6 \mathrm{ft}$. of distance from one another, washing hands often, and covering one's mouth and nose when interacting with others. Among several recommendations designed to reduce the spread of the disease, it is recommended that individuals engage in physical distancing, and this has resulted in a broader use of telehealth technology within the healthcare system (Wosik et al., 2020).

Although there may be numerous drivers of its utilization, telehealth allows for adherence to recommendations of physical distancing that are crucial to managing communicable diseases, like COVID-19 (Monaghesh \& 
Hajizadeh, 2020). High-risk patients, such as the immunocompromised or older adults, are protected from possible exposure when they seek medical care. Telehealth can minimize exposure of sick patients to healthcare staff, maintain personal protective equipment supply, and decrease the number of patients crowding in the facility. Typical services may include non-emergent care for chronic conditions, mental health services, or follow-up contact after hospitalization. Generally, there is support for the efficacy of telehealth care interventions (e.g., Chen et al., 2017; Dario et al., 2017; Marx et al., 2018; Rush et al., 2018; Shigekawa et al., 2018).

Despite its efficacy, numerous barriers to implementation have been identified. Common barriers include technological issues (e.g., low bandwidth/poor high-speed internet), cost and reimbursement, incompatible electronic health records and concerns about use with specific patient populations (e.g., homeless, highly complex patients; Lin et al., 2018). Previous barriers to implementation of telehealth that have been identified may have shifted with the rapid expansion and use of telehealth with COVID-19.

Most studies that examine barriers to use of telehealth do not examine provider self-efficacy, or confidence to use the equipment (e.g., Moore et al., 2017; Perry et al., 2020). Selfefficacy refers to an individual's perceived ability or belief that they can engage in a behavior that will achieve a specific goal (Bandura, 1978, 1994). Self-Efficacy Theory suggests that if there is a lack of self-efficacy, there is reduced incentive or motivation to engage in the relevant behavior and/or persevere when there are difficulties. Thus, in the context of rapid changes and implementation during telehealth care, one might posit that those who have greater self-efficacy or confidence in their use of telehealth may also have greater usage of services that meet physical distancing guidelines, and perseverance during the inevitable challenges that arise with rapid change.

Understanding providers' perceptions of telehealth usage in rural communities may help other communities understand barriers and concerns related to implementation, during and post-pandemic. This study aimed to (a) examine rates of telemedicine use among rural providers, (b) determine whether changes in telehealth use in this group were associated with provider confidence and perceived usefulness of technology, (c) compare these providers' perceptions of the "usefulness" of technology prior to and during the COVID-19 pandemic, and (d) examine barriers to implementation and use of telehealth within a rural sample. It was hypothesized that providers would report a significant increase in use of telehealth and increased beliefs that technology is useful during the pandemic compared to prepandemic. In addition, it was expected that greater increases in telehealth use would be associated with greater perceived usefulness of technology.

\section{Method}

Six hundred eighty-six providers were invited to complete an anonymous survey via Google Forms (C) on March 30, 2020, 10 days following administrative directives to cancel routine care appointments across the organization. Participants included 136 rural medical providers (19.8\% response rate) working at a rural Pennsylvania teaching hospital and associated satellite clinics. Locations associated with the hospital and satellite clinics were non-urban areas, as defined by U.S. Health Resources and Services Administration. Respondents were asked whether they worked in a rural site, to further ensure that they worked with a rural population. All methods and procedures were approved by the organization's institutional review board and human subjects committee. Prompts to complete the survey were sent 1 and 2 weeks later. The survey included questions about demographic information, previous experience using telehealth, and perceived changes in use of telehealth for patient care (percentage of patient visits using telehealth before and after COVID-19). It also included the Perceived Usefulness of Technology Scale (Davis, 1989) and questions to identify barriers that prohibited the use of telehealth.

Respondents completed the Perceived Usefulness of Technology Questionnaire (PUT; (Davis, 1989). The PUT is a six-item scale that examines respondents' perceived usefulness of technology. Respondents assess their beliefs about technology from 1 (Strongly disagree) to 7 (strongly agree) for items like, "I feel that electronic devices enhance my effectiveness on the job," and "Using electronic devices would improve my job performance." This scale had good reliability, $\alpha=0.95$.

Respondents selected barriers to telehealth from a checklist of options generated from previous literature (Lin et al., 2018). Providers were also permitted to identify other barriers in an open-ended fashion.

A different survey had been sent to the same group of providers 18 months prior, examining medical providers perceived usefulness of technology and other use of technology (see Terry \& Mathews, in press). Average PUT scores from that sample $(N=151,24 \%$ response rate) were compared to those of the current sample.

\section{Results}

Respondents included 136 medical providers who were an average of $46.5(S D=11.2)$ years old, primarily female $(52.2 \%)$, and identified as white $(84.2 \%)$, followed by Asian (11.3\%). Most medical providers indicated that they 
Table 1 Correlations of study variables $(N=136)$

\begin{tabular}{lllll}
\hline Variable & 1 & 2 & 3 & 4 \\
\hline 1. \% Change in telehealth use & - & & \\
2. Usefulness of technology (PUT) & 0.13 & - & \\
3. Confidence in telehealth abilities & $0.34^{*}$ & $0.27^{*}$ & - & \\
4. Negative belief about telehealth & 0.02 & $-0.40^{*}$ & $-0.25^{*}$ & - \\
\hline
\end{tabular}

${ }^{*}$ Correlation is significant at the $<.01$ level

were an MD or DO (58.1\%) or a nurse practitioner (25\%). The representation of medical providers who responded to the survey was similar to the overall organization $(58.5 \%$ MD/DO). Providers were from a variety of different specialties and subspecialties including family and internal medicine $(40.5 \%, n=87)$, surgery $(8.8 \%, n=19)$, and radiology $(5.1 \%, n=11)$. A total of 23 other specialties were identified and ranged from $0.5 \%$ of the sample to $4.2 \%$. No resident physicians completed the survey. Most medical providers $(86 \%)$ reported that they did not have experience using telehealth or virtual technology for patient encounters prior to the COVID-19 pandemic.

Providers reported that their use of telehealth care increased significantly following the pandemic, $(t$ $(132)=11.56, p=<0.01)$. Specifically, providers reported that the percentage of their virtual encounters increased from an average of $1.82 \%(S D=12.32)$ to $35.76 \%(S D=34.73)$.

Correlational analyses were conducted to examine associations between provider confidence in their ability to appropriately use technology, usage of telehealth, and their overall perceived usefulness of technology. Results suggested that the change in use of telehealth was significantly positively associated with providers' confidence in their abilities $(r=0.34, p<0.01)$. Confidence was also positively associated with perceived usefulness of technology,
( $r=0.40, p<0.01$ ), such that those who were more confident in their abilities also believed that technology was more useful in their professional lives. Finally, the belief that use of telehealth reduced the quality of patient care was negatively associated with confidence in one's ability $(r=-0.25$, $p=0.003)$ and their perceived usefulness of technology $(r=-0.40, p<0.001)$. See Table 1 for correlation matrix.

An independent-sample $t$-test was used to examine whether average PUT scores differed between the pre-pandemic and pandemic survey samples $(M=30.27, S D=9.31$ and $M=32.04, S D=7.78$, respectively). There was no significant difference in scores on the PUT before and after the pandemic, $t(276)=-1.71, p=0.09$.

Finally, respondents endorsed barriers to providing telehealth care. Figure 1 includes a list of barriers. Nearly three quarters of medical providers indicated that patients' need for a physical examination interfered with their use of telehealth. Other common barriers endorsed included a lack of technological literacy (72.6\%), patients' lack of Internet access $(65.9 \%)$, and patient complexity.

\section{Discussion}

Prior to the current COVID-19 pandemic, an estimated 15\% of family physicians across the United States were using telehealth in their everyday practice (Moore et al., 2017). Findings from our study suggest significant increase (34\%) in use during COVID-19 among rural medical professionals of varying specialties in a northeastern medical organization. Healthcare providers who were more confident with using telehealth services also found it to be more useful, whereas providers who associated telehealth with reduced quality of care tended to find it less useful. Interestingly, the overall perceived usefulness of telehealth did not significantly
Fig. 1 Barriers to use of telehealth

\section{Barriers to Use of Telehealth}

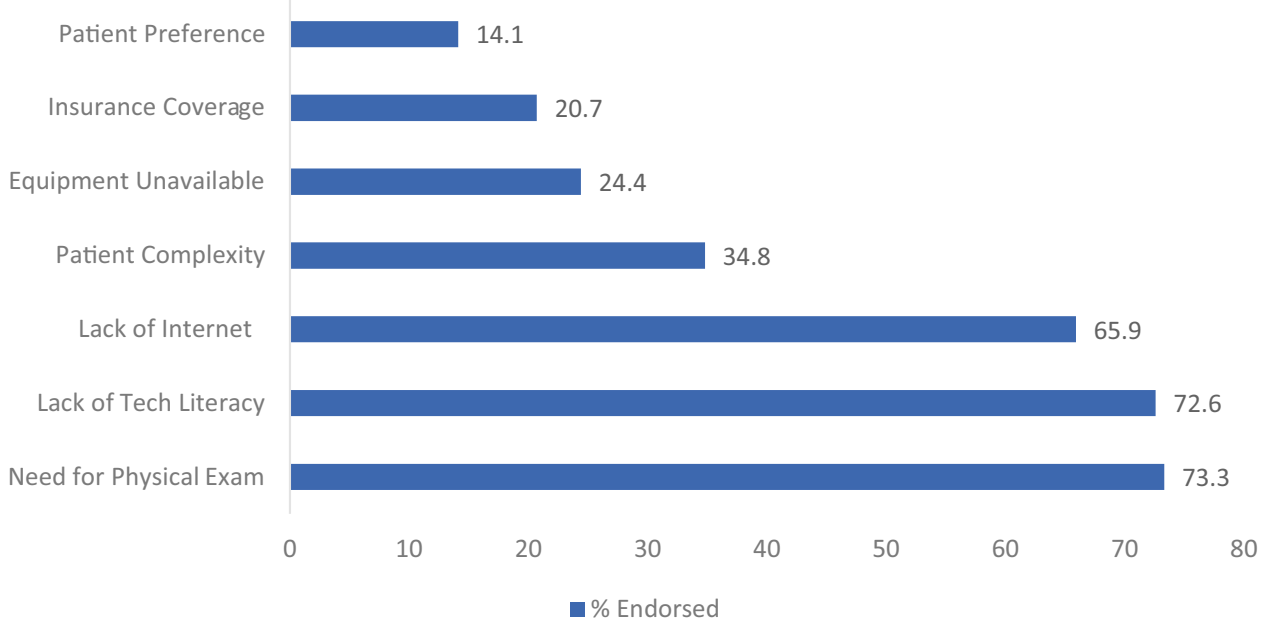


change pre- and post-COVID. Furthermore, surveyed providers most frequently cited the need for physical exams, concerns about patients' technological literacy, and/or problems with connectivity as barriers to telehealth. While the hypotheses of our study were partially supported, our general findings are consistent with other studies (Smith et al., 2020). That is, providers did report a greater utilization of telehealth care, and those that did also reported greater confidence in their abilities to use it.

It is not surprising that the use of telehealth has increased significantly since the onset of the pandemic, especially considering the difficulties of clinical access and financial implications (Farooq \& Ali, 2020). However, it is notable that the sample had limited experience using telehealth with patients prior to the pandemic, and those that were more confident in their abilities also reported greater use of telehealth in their practice. Previous studies have suggested that less than $1 \%$ of people in rural settings had experience using telehealth (Smith et al., 2020), although rural physicians are more likely to have used telehealth than urban counterparts (Jetty et al., 2017). It will be of interest to monitor longterm changes in telehealth utilization, even after the intense demand associated with the pandemic has receded.

The association between self-confidence or efficacy and telehealth use may be important for several reasons. Selfefficacy is ones' belief that s/he can succeed in a task, such as using a new telehealth platform for patient care. According to Social Cognitive Theory (Bandura, 1978), those with greater self-efficacy are more likely to approach challenges and complete them. Devising ways to increase self-confidence and efficacy for use of telehealth among providers might be an additional way to increase telehealth use. Given the correlational nature of this study, it is impossible to determine whether exposure to telehealth increased providers' confidence. For example, if certain departments were able to easily access and implement telehealth compared to others (i.e., dermatology versus emergency room), increased exposure to telehealth may have increased providers' self-efficacy. In addition, it may also be the case that providers who enjoy use of telehealth and/or believe it is more valuable may be reporting greater use. Future research might examine whether, consistent with this theory, greater self-efficacy results in greater usage of telehealth, or whether confidence increases due to the increased use of telehealth platforms. Designing studies to examine temporal relationships may aid administrators who need to rapidly disseminate and implement changes within organizations.

The concept of telehealth is not a novel one. Telehealth has been utilized since the early 2000s when various government agencies sought its use in disaster/emergency situations (Smith et al., 2020). It has been identified as a viable means of providing physically distanced access to medical care, which is crucial in limiting the spread of COVID-19.
However, until this current public health emergency, the uptake of telemedicine in everyday practice has been slow (Smith et al., 2020). Studies have demonstrated that a lack of clinician understanding and acceptance is a key factor in the limited everyday use of telehealth (Wade et al., 2014). Furthermore, prior to COVID-19, many healthcare providers have had limited exposure to telehealth and do not feel comfortable with it (Ayatollahi et al., 2015).

Researchers have identified significant barriers to workflow of providers using video and mobile health. Previously, barriers related to using video have been synthesized into three primary categories: (1) personal barriers, (2) clinical workflow and technology barriers, and (3) licensure, credentialing and reimbursement (Cowan et al., 2019). Examples of personal barriers might include concerns about being able to adequately establish rapport with patients or assess for non-verbal signs of psychiatric illness. This might be handled by training providers, allowing providers to observe others using this technology. For those using mobile health technology, one review identified eight social and organizational factors that impacted adoption of technology. These included workflow-related, patient-related, policy and regulations, culture/attitude or social influence, monetary factors, evidence-base, awareness, and user engagement (Jacob et al., 2020). Obstacles identified from this study highlight additional provider concerns about patient-based limitations, in addition to those previously identified (i.e., insurance).

Future research in rural health settings might explore other salient barriers related to dissemination of telehealth services. These studies might also include barriers reported by relevant support staff, like nurses or administrative staff. Understanding and identifying ways in which telehealth can be disseminated effectively and efficiently aligns with protective health behaviors (i.e., physical distancing). Given the increased use of telehealth in medicine, understanding the perceived advantages and disadvantages might provide clues to how organizations might better implement and use technology. As these barriers are further identified, and the specific impacts of these barriers are assessed, organizations could ultimately consider changes in policy and practices.

There were several limitations in this study. Findings from this study may not apply to other geographical areas, especially as the technological landscape shifts with availability of resources and escalation of disease. Future research with more representative samples might examine whether specialties that were already using telehealth (i.e., neurology, telepsych) had different experiences and perceptions of usefulness of technology. As noted, this study did not include support staff like nurses, who may be integral to "rooming" patients as they wait online for their medical provider.

Response rates to this survey were relatively low, and there was no way to connect responses from the pre-pandemic survey. In other words, although the survey was sent to the same 
provider group, it is possible that a different set of providers within the group responded and may have included those that were differentially impacted by the pandemic. In addition, given that this was on online survey, those with more positive experiences and greater technological self-efficacy may have been more inclined to respond.

\section{References}

Ayatollahi, H., Sarabi, F. Z. P., \& Langarizadeh, M. (2015). Clinicians' knowledge and perception of telemedicine technology. Perspectives in Health Information Management, 12, 1c.

Bandura, A. (1978). Self-efficacy: Toward a unifying theory of behavioral change. Perceived Self-Efficacy: Analyses of Bandura's Theory of Behavioural Change, 1(4), 139-161. https://doi.org/ 10.1016/0146-6402(78)90002-4

Bandura, A. (1994). Self-efficacy. In Encyclopedia of human behavior (Vol. 4, pp. 71-81). New York: Academic Press.

Centers for Disease Control and Prevention. (2020). Cases and deaths in the U.S. https://www.cdc.gov/coronavirus/2019-ncov/casesupdates/us-cases-deaths.html

Chen, Y.-Y., Guan, B.-S., Li, Z.-K., \& Li, X.-Y. (2017). Effect of telehealth intervention on breast cancer patients' quality of life and psychological outcomes: A meta-analysis. Journal of Telemedicine and Telecare, 24(3), 157-167. https://doi.org/10.1177/1357633X16686777

Cowan, K. E., McKean, A. J., Gentry, M. T., \& Hilty, D. M. (2019). Barriers to use of telepsychiatry: Clinicians as gatekeepers. Mayo Clinic Proceedings, 94(12), 2510-2523. https://doi.org/10.1016/j. mayocp.2019.04.018

Dario, A. B., Moreti Cabral, A., Almeida, L., Ferreira, M. L., Refshauge, K., Simic, M., Pappas, E., \& Ferreira, P. H. (2017). Effectiveness of telehealth-based interventions in the management of non-specific low back pain: A systematic review with meta-analysis. The Spine Journal, 17(9), 1342-1351. https://doi.org/10.1016/j.spinee.2017. 04.008

Davis, F. D. (1989). Perceived usefulness, perceived ease of use, and user acceptance of information technology. MIS Quarterly, 13(3), 319-340. https://doi.org/10.2307/249008

Farooq, I., \& Ali, S. (2020). COVID-19 outbreak and its monetary implications for dental practices, hospitals and healthcare workers. Postgraduate Medical Journal, 96, 791-792.

C Jacob A Sanchez-Vazquez C Ivory 2020 Social, organizational, and technological factors impacting clinicians' adoption of Mobile Health tools: Systematic literature review JMIR mHealth and uHealth 82 e15935 https://doi.org/10.2196/15935

Jetty, A., Moore, M., Coffman, M., \& Petterson, S. (2017). Rural family physicians are twice as likely to use telehealth as urban family physicians. Telemedicine and E-Health, 24(4), 268-276. https:// doi.org/10.1089/tmj.2017.0161
Lin, C. C., Dievler, A., Robbins, C., Sripipatana, A., Quinn, M., \& Nair, S. (2018). Telehealth in health centers: Key adoption factors, barriers, and opportunities. Health Affairs, 37(12), 1967-1974. https://doi.org/10.1377/hlthaff.2018.05125

Marx, W., Kelly, J. T., Crichton, M., Craven, D., Collins, J., Mackay, H., Isenring, E., \& Marshall, S. (2018). Is telehealth effective in managing malnutrition in community-dwelling older adults? A systematic review and meta-analysis. Maturitas, 111, 31-46. https://doi.org/10.1016/j.maturitas.2018.02.012

Monaghesh, E., \& Hajizadeh, A. (2020). The role of telehealth during COVID-19 outbreak: A systematic review based on current evidence. Research Square. https://doi.org/10.21203/rs.3.rs-23906/v1

Moore, M. A., Coffman, M., Jetty, A., Klink, K., Petterson, S., \& Bazemore, A. (2017). Family physicians report considerable interest in, but limited use of, telehealth services. Journal of the American Board of Family Medicine, 30(3), 320-330. https://doi. org/10.3122/jabfm.2017.03.160201

Perry, K., Gold, S., \& Shearer, E. M. (2020). Identifying and addressing mental health providers' perceived barriers to clinical video telehealth utilization. Journal of Clinical Psychology, 76(6), 1125-1134. https://doi.org/10.1002/jclp.22770

Rush, K. L., Hatt, L., Janke, R., Burton, L., Ferrier, M., \& Tetrault, M. (2018). The efficacy of telehealth delivered educational approaches for patients with chronic diseases: A systematic review. Patient Education and Counseling, 101(8), 1310-1321. https://doi.org/10.1016/j.pec.2018.02.006

Shigekawa, E., Fix, M., Corbett, G., Roby, D. H., \& Coffman, J. (2018). The current state of telehealth evidence: A rapid review. Health Affairs, 37(12), 1975-1982.

Smith, A. C., Thomas, E., Snoswell, C. L., Haydon, H., Mehrotra, A., Clemensen, J., \& Caffery, L. J. (2020). Telehealth for global emergencies: Implications for coronavirus disease 2019 (COVID-19). Journal of Telemedicine and Telecare, 26(5), 309-313. https://doi. org/10.1177/1357633X20916567

Terry, D. L., \& Mathews, D. (in press). Technology-assisted supplemental work among rural medical providers: Impact on burnout, stress, and job satisfaction. Journal of Healthcare Management.

Wade, V. A., Eliott, J. A., \& Hiller, J. E. (2014). Clinician acceptance is the key factor for sustainable telehealth services. Qualitative Health Research, 24(5), 682-694. https://doi.org/10.1177/ 1049732314528809

Wosik, J., Fudim, M., Cameron, B., Gellad, Z. F., Cho, A., Phinney, D., Curtis, S., Roman, M., Poon, E. G., Ferranti, J., Katz, J. N., \& Tcheng, J. (2020). Telehealth transformation: COVID-19 and the rise of virtual care. Journal of the American Medical Informatics Association, 27(6), 957-962. https://doi.org/10.1093/jamia/ ocaa067

Publisher's Note Springer Nature remains neutral with regard to jurisdictional claims in published maps and institutional affiliations. 